\title{
OCCURRENCE OF TRANSMITTED HIV-1 DRUG RESISTANCE AMONG DRUG-NAÏVE PREGNANT WOMEN IN SELECTED HIV-CARE CENTRES IN GHANA
}

\author{
Alexander Martin-Odoom ${ }^{1}$, Theophilus Adiku ${ }^{2}$, Elena Delgado ${ }^{3}$, Margaret Lartey ${ }^{4}$ and William K. \\ Ampofo ${ }^{5}$
}

Ghana Med J 2017; 51(1): 20-23～DOI: http://dx.doi.org/10.4314/gmj.v51i1.4

${ }^{1}$ Department of Medical Laboratory Sciences, School of Biomedical \& Allied Health Sciences, College of Health Sciences, University of Ghana, ${ }^{2}$ Department of Medical Microbiology, School of Biomedical and Allied Health Sciences, College of Health Sciences, University of Ghana, ${ }^{3}$ Centro Nacional de Microbiología, Department, Patogenia Viral/Uni of Biology and Variability of HIV, Instituto de Salud Carlos III, Ctra. de Majadahonda a Pozuelo, Km. 228220 Majadahonda (Madrid) Spain, ${ }^{4}$ Department of Medicine, School of Medicine and Dentistry, College of Health Sciences, University of Ghana, ${ }^{5}$ Department of Virology, Noguchi Memorial Institute for Medical Research, College of Health Sciences, University of Ghana, Legon, Ghana.

Corresponding author: Alexander Martin-Odoom

E-mail: siel.miel@yahoo.com

Conflict of interest: None declared

\section{SUMMARY}

Background: Access to antiretroviral therapy in Ghana has been scaled up across the country over the last decade .This study sought to determine the occurrence of transmitted HIV-1 drug resistance in pregnant HIV-1 positive women yet to initiate antiretroviral therapy at selected HIV Care Centres in Ghana.

Methods: Plasma specimens from twenty-six (26) HIV seropositive pregnant women who were less than 28weeks pregnant with their first pregnancy and ART naïve were collected from selected HIV care centres in three (3) regions in Ghana. Genotypic testing was done for the reverse transcriptase gene and the sequences generated were analyzed for HIV-1 drug resistance mutations using the Stanford University HIV Drug Resistance Database.

Results: Resistance mutations associated with the reverse transcriptase gene were detected in $4(15.4 \%)$ of the participants. At least one major drug resistance mutation in the reverse transcriptase gene was found in $3(11.5 \%)$ of the women.

Conclusion: The detection of transmitted HIV-1 drug resistance in this drug-naïve group in two regional HIV care sites is an indication of the need for renewed action in monitoring the emergence of transmitted HIV-1 drug resistance in Ghana.

Funding: None declared

Keywords: antiretroviral therapy, emergence, drug resistance, mutations

\section{INTRODUCTION}

Mother-To-Child-Transmission (MTCT) of HIV occurs when an HIV-infected pregnant woman passes on the virus to the baby during the pregnancy, labour, delivery or breastfeeding. Globally, the majority of the HIVinfected children under 5years were infected through MTCT. ${ }^{1}$ Interventions to prevent the transmission of HIV infection from a pregnant woman to her unborn baby involve a multi-pronged strategy which includes the use of antiretroviral drugs. ${ }^{2}$ Efforts to prevent MTCT in Ghana have evolved over the years from an era of single dose nevirapine (sd NVP) at the onset of labour (2003 to 2007) to a combination therapy initiated at 28 weeks of gestation coupled with an NVP at onset of labour. ${ }^{3}$ Since 2011 , combination therapy was initiated at 14 weeks of gestation. These efforts have led to a steady reduction in new HIV infections among children from 0 to 14 years. ${ }^{4}$

Viral resistance to antiretroviral therapy (ART) can emerge through selective pressure from antiretrovirals (ARVs), ${ }^{5}$ spontaneously generated polymorphisms in the viral genome in rare cases or acquired by infection with an already resistant virus. ${ }^{6}$ Transmitted HIV-1 Drug Resistance (tHIV-1DR) occurs when an individual is infected with an HIV-1 strain harbouring major drug resistant mutations. 
This reduces the effectiveness of ARVs against the virus and leads to expedited treatment failure, limiting the available drug options .6,7 Globally, as access to antiretroviral therapy (ART) was increased, the prevalence of transmitted HIV-1 drug-resistance increased from $2.8 \%$ before 2001 to $5.3 \%$ after 2003 in African countries. ${ }^{8}$ Results of studies in the United States of America and Europe have shown increases in the incidence of transmitted drug resistance mutations with time. ${ }^{6}$ In Ghana, data from HIV-1 drug resistance threshold survey done in 2009 showed low level $(<5 \%)$ prevalence of transmitted HIV-1 drug resistance (tHIVDR) in the Eastern region of Ghana, the first region to start ART in Ghana and the region with the highest HIV prevalence. ${ }^{9,10}$

The publication by Bonney and colleagues in $2013^{\mathbf{1 0}}$ recommended a repetition of the survey at same study sites after 2 years and extension to other sites for continuous monitoring of tHIVDR. However, this is yet to be implemented. As part of efforts to prevent MTCT, HIV positive pregnant women at the time of the study, were given combination antiretroviral therapy at 28 gestation weeks. This therapy will not provide optimum benefits to women infected with drug-resistant strains. It is therefore imperative to investigate the presence of drug resistance mutations in these pregnant women before their initiation on antiretroviral therapy.

This study sought to determine the occurrence of transmitted HIV-1 drug resistance mutations among ARTnaïve pregnant women at selected HIV Care Centres in three regions of Ghana.

\section{METHODS}

Study Design \& Population

This was a cross-sectional study carried out among HIV-infected patients who had been newly diagnosed at selected HIV Care and Support Centres in three regions in Ghana- Greater Accra, Ashanti and the Eastern regions. The study sites were the Korle - Bu Teaching Hospital in Accra and Tema General Hospital (both in the Greater Accra Region); Kumasi South Hospital, Suntreso Government Hospital, Kumasi, Bomso Clinic, Kumasi and Animwah Medical Centre, Kumasi (all four in the Ashanti Region); and the Eastern Regional Hospital, Koforidua (in the Eastern Region).

These selected study sites are health facilities that had all participated in the national Prevention of Mother-ToChild Transmission (PMTCT) of HIV programme for at least three (3) years and had data available and accessible. For each study site the electronic database on HIV positive patients was accessed with the consent of each health facility (study site).
Records of HIV positive pregnant women of less than 28 gestational weeks, who were yet to be initiated onto the prevention of mother-to-child transmission (PMTCT) of HIV programme and were drug-naïve, were accessed. Contact details of such women who satisfied the criteria were extracted from the database from the chosen sites. After one year sample collection period, 26 of such women were traceable and accessible and consented to participate in the study. After these women agreed to participate, they were duly enrolled and the structured questionnaires were administered to obtain the demographic information from them to augment the data extracted from the hospital folders.

The convenience sampling technique was thus used enrolling participants. The number of participants enrolled (26) comprised nine (9), ten (10) and seven (7) from the selected sites in Greater Accra, Eastern and Ashanti regions respectively. For the purposes of the study, it was affirmed that these women had not had any prior exposure to antiretroviral.

\section{Sample Collection and Processing}

A structured questionnaire was used to obtain basic socio-demographic and clinical data from the patients who had consented to participate in the study.

Ten (10) mls of whole blood was taken from the antecubital vein of each participant and distributed into EDTA- treated tubes (9mls) and CD4 tubes $(1 \mathrm{ml})$. One $\mathrm{ml}$ of the whole blood was pipetted into a CD4 tube for the CD4 $+\mathrm{T}$ cell count measurement on site, since this was done routinely at these sites, by flow cytometry using BD FACSCount Cytofluorometer and cell-quest software (Becton-Dickinson, Franklin Lakes, New Jersey, USA).

The blood samples in the EDTA- treated tubes $(9 \mathrm{mls})$ were transported in cool boxes with ice packs to the Virology Department of Noguchi Memorial Institute of Medical Research (NMIMR) at Legon, Accra, Ghana. At the NMIMR, plasma was separated from the whole blood through centrifugation at $2000 \mathrm{~g}$ for 10 minutes and stored in vials at $-70^{\circ} \mathrm{C}$ until further laboratory analysis.

\section{HIV Typing}

HIV typing of the samples was done using a line immunoblot assay, INNO-LIA HIV I/II Score kit (by INNOGENETICS, BELGIUM). The manufacturer's instructions for preparing the reagents, carrying out the test procedure and applying the interpretation criteria were followed to differentiate HIV-1 from HIV-2 infections. Samples confirmed as HIV-1 were further analyzed by genotyping. 


\section{Genotyping}

Viral ribonucleic acid (RNA) was extracted from $140 \mu 1$ of plasma using the QIAamp Viral RNA Kit (QIAGEN, $\mathrm{GmbH}$, Germany) according to the manufacturer's instructions. The extract was eluted in $60 \mu 1$ of elution buffer. Each RNA extract was subjected to RT-PCR and nested PCR to amplify the reverse transcriptase (RT) gene of HIV-1. QIAGEN One-Step RT-PCR Kit (QIAGEN, GmbH, Germany) and AmpliTaq ${ }^{(\mathrm{R})}$ Gold Master Mix reagents (Applied Biosystems, USA) were used for the amplification according to the manufacturer's protocol. ${ }^{11}$

Primers used were DRRT1L (sense) and DRRT4L (antisense) for the RT-PCR and DRRT7L(sense) and DRRT6L (antisense) for the nested PCR as previously described $^{12}$ with modifications in thermal conditions as described previously.$^{\mathbf{1 3}}$ Nested PCR products were analyzed by $1.5 \%$ agarose gel electrophoresis in $1 \mathrm{X}$ Tris Acetate EDTA (TAE) buffer. The positive PCR products were purified using QIAquick PCR purification kit (QIAGEN, GmbH, Germany) and sequenced using Big Dye Terminator Cycle Sequencing kit vs. 3.1 (Applied Biosystems, USA) in 3130 Genetic Analyzer.

The Align IR version 2.0 software (from LI-COR Inc., Michigan Technology University, 2001) was used for assembling, editing and alignment of raw sequence data. The edited sequences were FASTA-formatted and submitted to the Stanford University HIV Database Programme $^{14}$ (http://hivdb.stanford.edu) to generate and interpret drug resistance mutation data and also assign subtypes. Resistance associated mutations were considered for analysis with regards to the 2013 IAS-USA recognized mutations for NRTIs and NNRTIs.$^{15}$

\section{RESULTS}

Types of HIV Infection

Twenty-five (25) out of the 26 patients analyzed (96.2\%), were infected with HIV Type 1. HIV Type 2 infection was not found in this study though one participant $(3.8 \%)$ was infected by both HIV $1 \& 2$ (dual infection). The trend is shown in Table 1.

Table 1 HIV Type Variations

\begin{tabular}{ll}
\multicolumn{1}{c}{ HIV SUBTYPES } & NUMBER N (\%) \\
\hline HIV-1 & $25(96.2)$ \\
HIV-2 & $0(0)$ \\
HIV-1/2 & $1(3.8)$ \\
TOTAL & $26(100)$ \\
\hline
\end{tabular}

HIV-1 Drug Resistance Associated Mutations

Out of the 26 samples analyzed, 18(69.2\%) were successfully amplified and sequenced. Four samples (15.4\%) had drug resistance associated mutations (DRAMs). Major and minor resistance mutations were identified according to the March 2013 edition of the
IAS-USA drug resistance mutations updated list ${ }^{15}$ Three samples had resistance mutations to both NRTIs and NNRTIs. While two of these patients had major mutations to both classes of drugs, the third had only minor mutations to both drug classes (Table 2). Major NRTI mutations, M184V and L210W, and a minor NRTIs mutation, V75S, were seen in this study. For NNRTIs; K103N, V106A and E138A were the major mutations seen and $\mathrm{A} 98 \mathrm{G}$ was the minor mutation detected.

Table 2 HIV-1 Reverse Transcriptase Drug Resistance Mutations in ART-naïve patients

\begin{tabular}{|c|c|c|c|c|c|}
\hline \multirow[t]{2}{*}{$\begin{array}{c}\text { SAMPLE } \\
\text { ID }\end{array}$} & $\begin{array}{l}\text { SITE } \\
\text { (REGION) }\end{array}$ & $\begin{array}{l}\text { MAJOR } \\
\text { TIONS }\end{array}$ & MUTA- & $\begin{array}{l}\text { MINOR } \\
\text { TIONS }\end{array}$ & MUTA- \\
\hline & & NRTIs & NNRTIs & NRTIs & NNRTIs \\
\hline KDC1/6 & EASTERN & M184V & $\mathrm{K} 103 \mathrm{~N}$ & - & - \\
\hline KDC1/10 & EASTERN & L210W & V106A & - & - \\
\hline KDC1/1 & EASTERN & - & - & - & A98G \\
\hline $\mathrm{KBC1/5}$ & $\begin{array}{l}\text { GREATER } \\
\text { ACCRA }\end{array}$ & - & E138A & V75S & - \\
\hline
\end{tabular}

Three (3) patients among the study group had detectable viral loads; these were $\mathrm{KBC} 1 / 3, \mathrm{KDC} 1 / 5$ and $\mathrm{KDC} 1 / 9$ who had 444 copies $/ \mathrm{ml}, 933 \mathrm{copies} / \mathrm{ml}$ and $692 \mathrm{copies} / \mathrm{ml}$ respectively. All the four (4) samples that had drug resistance mutations had viral loads below the assay detection limit of 153 copies $/ \mathrm{ml}$.

\section{DISCUSSION}

The type distribution of HIV found in this study is similar to that reported in the HIV Sentinel Survey Reports for Ghana in 2011 where HIV-1 was $96.4 \%$, HIV-2 was $0.7 \%$ and HIV-1 \& 2 was $2.9 \%{ }^{16}$ The HIV Sentinel Survey Report has established the predominance of HIV-1 in Ghana and the continual co-circulation of HIV-2 although in lower proportions.

The presence of major drug resistance mutations in ART-naïve patients signals the possible transmission of drug-resistant strains. Transmitted drug resistance of HIV-1 (tHIVDR) occurs when previously uninfected individuals are infected with a drug-resistant variant of the virus.$^{6,17}$ A threshold study performed in the Eastern Region of Ghana in 2009 by Bonney and colleagues found major drug resistance mutations in one out of 47 samples sequentially collected, and classified the prevalence of tHIVDR in that population as low $(\leq 5 \%) .{ }^{10}$ Thus the presence of major mutations in three out of 26 drug-naïve samples could be an indication that transmitted drug resistance of HIV-1 (tHIVDR) is on the increase in Ghana. Two of the three samples that had the major mutations were collected from the Eastern Region (See Table 2). The Eastern Region of Ghana is the region where ART was first introduced into the country in $2003^{10}$ and so represents a population that has the longest experience with ART in Ghana. 
Although another study in the Eastern regional capital, Koforidua, in 2009, found no major resistance mutations in that ART-naïve group, ${ }^{\mathbf{1 8}}$ the results from this study and that of the first-ever threshold survey carried out in Ghana in two different sites in the Eastern Region 10 show an increasing trend. This calls for surveillance for tHIVDR in a larger study population in the eastern region and elsewhere in Ghana to further understand the extent of its spread and introduce appropriate measures to curb its devastating effects on the nation's antiretroviral therapy programme.

The presence of major drug resistance mutations among ART -naïve pregnant women also has the potential to offset the gains made in the programmes aimed at eliminating mother-to-child transmission of HIV.

\section{CONCLUSION}

The occurrence of major HIV-1 drug resistance mutations among drug-naïve HIV-1 positive pregnant women studied is an indication of the need to activate surveillance for the monitoring of possible increasing levels of transmitted HIV-1 drug resistance in Ghana.

\section{ACKNOWLEDGEMENT}

The authors are grateful to Dr. John Kofi Odoom of Noguchi Memorial Institute for Medical Research (NMIMR) for his immense help in the assemblying, editing and alignment of the raw sequence data; to Dr. Evelyn Yayra Bonney and Ms. Nana Afia Asante Ntim, both of NMIMR, for their immense contribution during the research.

\section{REFERENCES}

1. UNAIDS, (2013). UNAIDS Report on Global AIDS Epidemic, 2013.

2. Duerr, A., Hurst, S., Kourtis, A.P, Rutenberg, N., Jamieson, D.J. Integrating family planning and prevention of mother-to-child HIV transmission in resource-limited settings. The Lancet 2004;366: 261263.

3. National AIDS/STI Control Programme, Ghana (2010), An overview of HIV and AIDS in Ghana. Current Situation, Projections and Interventions.

4. National HIV Prevalence \& AIDS Estimates Reports, 2011-2015. National AIDS/STI Control Programme (NACP), Ghana Health Service, Ministry of Health, Accra, Ghana, March 2012.

5. Virco Interactive HIV Resistance Learning Module (2006). Available at:http://www.vircolab.com/hiveducational-forum/hivaids-learning modules.

6. Price, M.A., Wallis, C.L., Lakhi, S. et al, Transmitted HIV Type 1 Drug Resistance Among Individuals with Recent HIV Infection in East and Southern Africa. Aids Research and Human Retroviruses 2011;27(1) Rev 14: 17-27.
7. Booth, C. L. and Geretti, A.M. (2007).Prevalence and determinants of transmitted antiretroviral drug resistance in HIV-1 infection. $J$ Antimicrob Chemother 2007; 59 (6):1047-1056.

8. Frentz, D., Boucher, C.A.B., van de Vijver, D.A.M.C Temporal changes in the epidemiology of transmission of drug-resistant HIV-1 across the world. AIDS Rev. 2012; 14(1)17-27.

9. National HIV Prevalence \& AIDS Estimates Reports, 2013-2020. National AIDS/STI Control Programme (NACP), Ghana Health Service, Ministry of Health, Accra, Ghana, June, 2014.

10. Bonney, E. Y., Addo, N. A., Ntim, N. A. A., Ampofo, W. et al, 2013. Low level of transmitted HIV drug resistance at two HIV care centres in Ghana: A Threshold Survey. Ghana Med J 2013;47(2):

11. Handbook, QIAGEN One-Step RT-PCR Reagents, 2006.

12. Fujisaki, S., Ibe, S., Asagi, T et al. Performance and Quality Assurance of GenotypicDrug Resistant Testing for Human Immunodeficiency Virus type 1 in Japan. Jpn J. Infect Dis 2007;60:113-117.

13. Villahermosa, M.L., Thomson, M., Vazquez de Parga, E., Cuevas, M.T. et al. Improved conditions for extraction and amplification of human immunodeficiency virus type 1 RNA from plasma samples with low viral load. J.Hum Virol 2000;3(1): 27-34.

14. Stanford University HIV Drug Resistance Database, 2011. HIVdb: Genotypic Resistance Interpretation Algorithm; (www.hivdb.stanford.edu).

15. Johnson, V.A., Calvez, V.,Gunthard, H.F., Paredes, R., Pillay, D., Shafer, R.W., Wensing, A.M. and Richman, D.D (2013). Update of the Drug Resistance Mutations in HIV-1: March 2013. Top Antivir Med.2013; 21(1):6-14. 2013 IAS-USA.

16. HIV Sentinel Survey Report, 2011. National AIDS/STI Control Programme, Ghana Health Service, Ministry of Health, Accra, Ghana, 2012.

17. World Health Organization Programmatic Update, 2012.Use of Antiretroviral Drugs for treating pregnant women and preventing HIV infection in Infants. April, 2012.

18. Nii-Trebi, N.I., Ibe, S., Barnor, J.S., Ishikawa, K., Brandful, J.A.M., Ofori, S.B. et al HIV-1 DrugResistance Surveillance among TreatmentExperienced and -Nave Patients after the Implementation of Antiretroviral Therapy in Ghana. PLoS ONE 2013;8(8): e71972. 